$>$ L'un des cinq axes permettant de comparer des sociétés et des cultures est celui de « naître, vivre et mourir ». Avant de naître, un être humain doit avoir été conçu. Et ce sont précisément les représentations que se font les sociétés du processus de fabrication d'un enfant qui sont explorées dans cet article. Les données ethnographiques concernant vingt-six sociétés ont été comparées et le résultat de cette comparaison est que, dans aucune de ces sociétés, on ne pense qu'un homme et une femme suffisent à fabriquer un enfant, que cet enfant soit un humain ordinaire ou un humain extraordinaire, un chef ou un homme-dieu. Partout, quels que soient les systèmes de parenté ou les structures politicoreligieuses, ce que font un homme et une femme c'est fabriquer un fœtus, qui nécessitera, pour devenir un enfant humain complet, l'intervention d'agents plus puissants que les humains, c'est-à-dire des ancêtres ou des dieux. Dans cet article sont résumés les résultats de cette analyse comparée de quelques théories culturelles de la procréation et de la conception des enfants. <

\section{II faut toujours plus qu'un homme et une femme pour faire un enfant}

Maurice Godelier

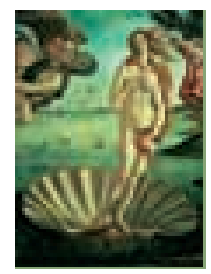

pour devenir un enfant. Ce qui manque souvent est ce qu'on appelle une âme, un esprit, mais c'est aussi le souffle, et parfois même le nez ou les doigts.

\section{Ce qu'il faut ajouter pour faire d'un fœtus un enfant}

Ces agents qui coopèrent avec les humains pour faire des enfants sont de deux sortes: des ancêtres ou des divinités; parfois, les deux sont nécessaires. Les ancêtres sont certes des humains, mais qui vivent une autre vie par-delà la mort et viennent parfois revivre dans le corps d'un enfant. Celui-ci est le plus souvent l'un de leurs descendants, mais ce peut aussi être le fils d'un voisin ou d'un ami, comme chez les Inuit. En général, cet ancêtre se manifeste à travers le nom donné à l'enfant et, habituellement, cet enfant ne garde rien en mémoire de toutes les existences, de toutes les expériences qu'ont vécues ceux ou celles qui avaient porté ce même nom avant lui. Dans beaucoup de sociétés, la naissance d'un être humain n'est pas un commencement absolu, de même que la mort n'est jamais la fin de la vie. Mais les ancêtres ne suffisent pas. II faut que des puissances qui ne relèvent pas de l'ordre humain interviennent, le Soleil chez les Baruya, Dieu chez les chrétiens, Sila chez les Inuit. Un enfant ne naît pas du seul jeu des unions sexuelles et des rapports de parenté, il s'insère en même temps dans une totalité cosmique et sociale qui déborde et englobe la sphère de la parenté. Dans toutes les sociétés étudiées, même dans les sociétés où le sperme n'entre pas dans la conception d'un 
enfant, l'apparition d'un fœtus dans le ventre d'une femme implique que l'homme et la femme se sont unis sexuellement. Que ce soit pour bloquer avec du sperme le sang menstruel, que ce soit pour ouvrir la voie à un enfant-esprit ou pour nourrir le fœtus, les hommes et les femmes doivent avoir des rapports sexuels et ils en attendent comme conséquence possible la naissance d'un enfant, quelle que soit la part réelle ou imaginaire que la société prête à telle ou telle substance masculine ou féminine dans la fabrication d'un enfant. Cette constatation met fin à la longue polémique qui a fait rage chez les anthropologues, depuis Malinowski jusqu'à Leach, à propos de l'ignorance des aborigènes australiens ou d'autres sociétés humaines sur le rôle des sexes dans la procréation. Chacun sait que pour les aborigènes australiens, comme pour les Trobriandais ou les $\mathrm{Na}$, les enfants existent avant même d'être conçus, sous forme d'enfant-esprit ou de fœtus déposés dans une matrice. Mais aucune société ne semble penser que des enfants naissent d'humains ordinaires sans rapports sexuels entre eux. En revanche, toutes les sociétés admettent que, dans certaines circonstances, des femmes peuvent être fécondées par des êtres surnaturels et donner naissance à des enfants sans avoir eu de contact avec un sexe d'homme; un exemple en est la naissance du Christ.

\section{Représentations imaginaires (pour nous) de la procréation}

Les représentations culturelles du processus de procréation des enfants sont des constructions imaginaires, et les pouvoirs donnés ou refusés aux substances du corps (sperme, sang menstruel...) ont un caractère fantasmatique: le sperme n'a jamais nourri aucun fœtus, le sang menstruel ne s'est jamais transformé par lui-même en fœtus. Mais les enjeux de ces représentations imaginaires ne sont ni imaginaires ni seulement symboliques. D'une part, elles légitiment les principes organisant les rapports de parenté et la transmission de biens, de statuts ou de pouvoirs par les hommes ou par les femmes, ou par les deux sexes selon que les systèmes de parenté sont patrilinéaires, matrilinéaires ${ }^{1}$, bilinéaires ou non-linéaires. D’autre part, elles inscrivent d'avance dans le corps de l'enfant les rapports de domination qui existent entre les sexes, ainsi que les rapports de force et de pouvoir - poli-

\footnotetext{
${ }^{1}$ Règle de filiation qui décide que l'individu acquerra les principaux éléments de son statut et notamment son appartenance à un groupe de parenté par référence aux seuls biens généalogiques passant par les femmes (ou par les hommes pour patrilinéaire)
}

tiques, économiques et rituels - qui existent entre les groupes qui composent une société.

Au nom du rôle primordial de l'homme dans la procréation, les femmes Baruya sont exclues de la propriété de la terre, de l'usage des armes, de l'accès au Soleil et aux divinités. Ces représentations sont des faits sociaux aux conséquences réelles. Pour dominer, il faut aussi disjoindre les corps des dominants et des dominés et en altérer les substances. L'homosexualité rituelle des Baruya réalise cette disjonction et cette transmutation. Les substances n'existant pas seules, à la survalorisation du sperme correspond la surdévalorisation du sang menstruel. On comprend que si les femmes Baruya sont elles-mêmes convaincues de porter en elles, dans leur corps, par leur sang, une menace dirigée contre les hommes et, à travers eux, contre l'ordre social et cosmique, elles peuvent se sentir responsables des désordres qui pourraient subvenir si elles ne géraient pas comme il se doit les éléments de leur corps qui peuvent se détacher d'elles et agresser les corps des autres. Mais, dans certaines circonstances, les dominés peuvent rompre l'étau idéel et social qui les amène à accepter la vie qu'ils vivent au nom de ce qu'ils sont. C'est ainsi que les femmes Telefolmin, converties au christianisme par les prédicateurs d'une secte protestante fondamentaliste qui annonçait le retour du Christ et le revival de l'humanité, ont été les premières en 1978 et 1979 à détruire les reliques qui étaient au cœur des rituels masculins dont elles étaient exclues.

\section{Le sexe au service de la société}

Si sexes et corps sexués sont utilisés pour fabriquer de la vie et de l'ordre social, voire cosmique, ils sont partout mis au service de multiples autres réalités, économiques, politiques, religieuses... Les rapports de parenté sont le lieu où s'exerce, dès la naissance et directement, le premier contrôle social de la sexualité des individus, aussi bien celle qui les pousse vers des personnes du sexe opposé que celle qui les attire vers des personnes du même sexe. Cette subordination de la sexualité individuelle n'est pas celle d'un sexe à l'autre, c'est la subordination d'un domaine de la vie sociale aux conditions de reproduction d'autres rapports sociaux. C'est la place de ce domaine à l'intérieur de la structure de la société. Cette subordination de la sexualité est le point de départ d'un mécanisme qui imprime dans la subjectivité la plus intime de chacun l'ordre ou les ordres qui règnent dans la société et qui doivent être respectés si celle-ci doit se reproduire. Cette empreinte se réalise par le jeu des représentations du corps et de la personne, et du rôle qu'on prête 
à chacun des sexes et à d'autres agents dans le processus qui donne naissance à un enfant, à la vie. C'est à travers ces représentations que se légitiment non seulement l'appropriation de l'enfant par des adultes considérés comme ses parents, mais également la place dans la société que son sexe lui réservera. À travers les représentations du corps sexué et de la fabrication des enfants, la sexualité se met non seulement à témoigner de l'ordre qui règne dans la société, mais à témoigner que cet ordre doit continuer à régner. Les corps, les sexes fonctionnent partout comme ces poupées ventriloques qu'on a du mal à faire taire et qui tiennent, à des interlocuteurs qu'elles ne voient pas, des discours qui ne viennent pas d'elles. La sexualité - comme ces poupées ventriloques - ne parle pas, on parle en elle, on parle par elle. Mais qui parle? Et pourquoi de là? Car c'est précisément dans la mesure où la sexualité est d'avance contrainte de servir de langage et de légitimer des réalités qui sont autres qu'elle, qu'elle devient source de fantasmes et d'univers imaginaires. Mais ce n'est pas la sexualité ici qui fantasme sur la société, c'est la société qui fantasme dans la sexualité. Ce n'est pas la sexualité qui aliène, c'est elle qui est aliénée.

Ces représentations fantasmatiques du corps sont la plupart du temps des idées et des images partagées par les deux sexes, qui résument et codent l'ordre social et inscrivent ses normes dans le corps de chacun. C'est ce partage des mêmes représentations et leur enfouissement dans le corps qui scellent en chaque individu, au-delà du langage, la pensée et la société, et qui font du corps une source d'évidences sociales et cosmiques. D'aliénée, la sexualité devient alors instrument d'aliénation. À la limite, une femme Baruya regardant le sang couler entre ses cuisses n'a plus rien à dire contre son sort, elle se sait et se vit coupable et, par là, responsable de ce qui lui arrive. De ce fait, on comprend pourquoi la sexualité est vécue comme quelque chose qui peut à tout moment questionner et subvertir l'ordre de la société et de l'univers. D'où les multiples tabous qui l'entourent.

\section{Le sexe machine désirante et machine ventriloque}

Les représentations du corps déterminent ainsi dans chaque société une sorte d'anneau de contraintes qui enserre l'individu, un anneau qui constitue la forme même, paradoxalement impersonnelle et anonyme, de son intimité. Et c'est dans cette forme anonyme de l'intimité à soi qui lui est imposée dès la naissance, et qui organise à l'avance ses rencontres avec l'autre, que l'enfant va commencer à vivre son désir d'autrui. Alors qu'il est déjà approprié par d'autres, ses parents, leur groupe social..., il va spontanément vouloir se les approprier. Et c'est là qu'il va découvrir qu'il ne peut tous se les approprier, que certains, père, mère, sœur, frère..., sont interdits à son désir. La sexualité «machine-désirante» s'oppose à elle-même «machine-ventriloque». Et finalement, même si un homme et une femme ne suffisent nulle part à faire un enfant, celui-ci ne peut cependant pas tourner vers eux son désir. Entre eux et lui se dresse le tabou de l'inceste, mais c'est une autre histoire... $\diamond$

One needs always more man and woman to make a child

\section{BIBLIOGRAPHIE}

- Godelier M. La production des grands hommes. Pouvoir et domination masculine chez les Baruya de Nouvelle-Guinée. Prix de l'Académie Française. Paris, Fayard, 1982. Traductions anglaise (Cambridge, CUP), allemande (Francfort, Campus), espagnole (Madrid, Akal), 1982.

- Godelier M, Panoff M. La production du corps. Approches anthropologiques et historiques. Paris: Éditions des Archives Contemporaines, 1998.

- Godelier M, Panoff M. Le corps humain, supplicié, possédé, cannibalisé. Paris: Éditions des Archives Contemporaines, 1998.

- Godelier M (entretien avec). L'imaginaire, le symbolique et le réel. Sciences Humaines 2001-2002; $n^{\circ} 35$ (hors série Les sciences de la cognition) : 20-2.

- Godelier M. Métamorphoses de la parenté. Paris: Fayard, 2004: 678 p.

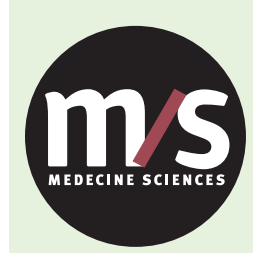

Tarifs d'abonnement M/S - 2005

Abonnez-vous

à Médecine/Sciences
$>$ 1985-2005, depuis 20 ans, grâce à $\mathrm{m} / \mathrm{s}$, vous vivez en direct les progrès des sciences biologiques et médicales

TIRÉS À PART

M. Godelier
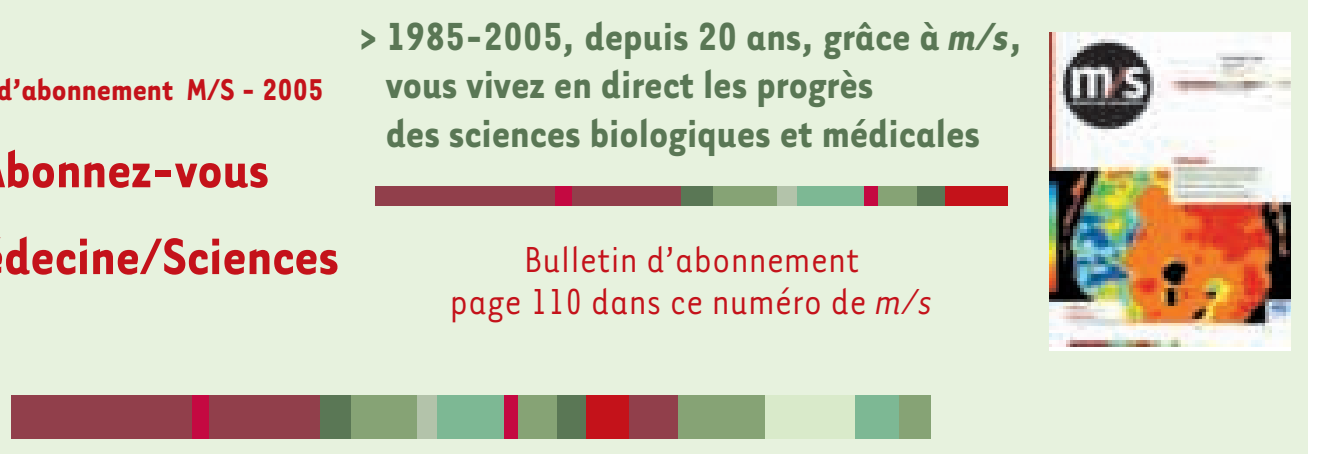